\title{
La integración curricular: Una experiencia en el primer nivel de diplomado de la carrera de Licenciatura en Pedagogía con énfasis en Educación Preescolar
}

\author{
Curriculum Integration: An Experience in the First Year of the \\ Two-year Program Conducive to a Licenciatura in Pedagogy with a \\ Specialization in Preschool Education
}

\author{
Ana Lucía Chaves Álvarez ${ }^{1}$ \\ Centro de Investigación y Docencia en Educación \\ Universidad Nacional \\ Heredia, Costa Rica \\ alchavesalvarez@hotmail.es \\ Dora Hernández Vargas ${ }^{2}$ \\ Centro de Investigación y Docencia en Educación \\ Universidad Nacional \\ Heredia, Costa Rica \\ dorahv@gmail.com \\ Jorge León Sánchez ${ }^{3}$ \\ Centro de Investigación y Docencia en Educación \\ Universidad Nacional \\ Heredia, Costa Rica \\ jorgeuna@gmail.com
}

\footnotetext{
Licenciada en Pedagogía con énfasis en Educación Preescolar (UNA). Experiencia docente en instituciones educativas costarricenses. Académica de la División de Educación Básica, del CIDE. Ha impartido diferentes cursos de la Carrera de Pedagogía con énfasis en Preescolar y ha trabajado como miembro del equipo del Proyecto de Acreditación de la División de Educación Básica, del Centro de Investigación y Docencia en Educación (CIDE), de la Universidad Nacional, así como en los procesos de Intervención Pedagógica del I Nivel de Diplomado de la Carrera de Pedagogía con énfasis en Educación Preescolar. Actualmente es estudiante de la Maestría en Pedagogía con énfasis en Desarrollo y Atención Integral de la Primera Infancia, de la Universidad Nacional y labora en la División de Educación Básica, Centro de Investigación y Docencia en Educación, Universidad Nacional,Heredia, Costa Rica.

2 Máster en pedagogía con énfasis en Diversidad en los Procesos Educativos y Licenciada en Pedagogía con énfasis en Educación Preescolar por la Universidad Nacional. Se ha desempeñado como maestra de música en el nivel preescolar y en primaria. Actualmente labora como profesora de la División de Educación Básica en el Centro de Investigación y Docencia en Educación en la carrera de Educación Preescolar.

3 Máster en Pedagogía con Énfasis en Atención a la Diversidad, Licenciado en Psicología, ambos por la Universidad Nacional de Costa Rica. Ex miembro del Consejo Universitario de la Universidad Nacional. Tiene experiencia en atención psicológica para la promoción de la salud, el desarrollo humano integral y educativo, así como en programas para el desarrollo del talento y altas capacidades en instituciones educativas. Actualmente, labora en la División de Educación Básica y la División de Educacion Rural del Centro de Investigación y Docencia en Educación (CIDE), de la Universidad Nacional, como académico de las carreras de Pedagogía con énfasis en Educación Preescolar, Pedagogía con énfasis en I y II Ciclo y Educación con énfasis en Educación Rural I y II Ciclos.
} 


\author{
Zulay Pereira Pérez ${ }^{4}$ \\ Centro de Investigación y Docencia en Educación \\ Universidad Nacional \\ Heredia, Costa Rica \\ zpereira@una.ac.cr \\ Marie Claire Vargas Dengo \\ Centro de Investigación y Docencia en Educación \\ Universidad Nacional \\ Heredia, Costa Rica \\ educare@una.ac.cr
}

Recibido 28 de setiembre de 2011 • Aceptado 24 de octubre de 2011

Resumen. Se plantea una reflexión acerca de la experiencia de integración curricular realizada por académicos y académicas universitarias, del primer nivel de diplomado de la carrera de Licenciatura en Pedagogía con énfasis en Educación Preescolar, de la División de Educación Básica de la Universidad Nacional, durante el primer semestre del año 2011. La integración ha sido concebida por el equipo del nivel como un proceso de crecimiento personal y profesional, para generar oportunidades que permitan el fortalecimiento de equipos docentes y de los cursos del nivel, en procura de brindar mayores oportunidades para el desarrollo estudiantil.

Palabras claves. Integración curricular, preescolar, académicos, educación superior.

Abstract. This paper is a reflection on the curriculum integration experience of faculty members in the first year of the two-year program conducive to a Licenciatura $(*)$ in Pedagogy with a specialization in Preschool Education of the Division of Basic Education, Universidad Nacional, Costa Rica, during the first half of 2011. The integration has been designed by the faculty members as a process of personal and professional growth, which will help to generate opportunities for team building, student development and integration of courses.

Keywords. Curriculum integration, Preschool, Academic, Faculty, Higher education.

\footnotetext{
Doctora en Educación, de la Universidad Estatal a Distancia, Costa Rica. Catedrática de la Universidad Nacional, Costa Rica. Máster y Licenciada en Psicología, de la Universidad de Costa Rica. Adscrita al Colegio Profesional de Psicólogos de Costa Rica. Ex coordinadora del Programa de Investigación en Epistemología Genética y Educación del IIMEC, actualmente Instituto de investigación en Educación (INIE) de la Universidad de Costa Rica (UCR). Ex Directora y docente de la División de Educación Básica del Centro de Investigación y Docencia en Educación de la Universidad Nacional, Costa Rica. Investigadora del Centro de Investigación y Docencia en Educación de la Universidad Nacional, Costa Rica. Tiene publicaciones en el campo de la Psicología y la Pedagogía.

5 Máster en Currículo e Instrucción con énfasis en Educación Especial Bilingüe de The George Washington University, USA. Doctoranda del Programa Latinoamericano de Doctorado en Educación, Universidad de Costa Rica. Académica de la División de Educación Básica del Centro de Investigación y Docencia en Educación (CIDE), Universidad Nacional, Costa Rica, donde ha formado parte del equipo de investigación del Proyecto Perfiles, dinámicas y desafíos de la educación costarricense y del Proyecto UNA educación de calidad que atiende a estudiantes con discapacidad en dicha institución. Tiene publicaciones en los campos de Pedagogía y de Educación Especial.
}

(*) Translator's Note: "Licenciatura" or "Licentiate's Degree" is a two year post-graduate study program, usually including thesis. 


\section{Introducción}

La Universidad Nacional (UNA), en Costa Rica, cuenta con un Modelo pedagógico que inicia su implementación, particularmente, a partir del 2008. Dicho modelo pretende, mediante “(...) la actualización profesional y la innovación curricular, potenciar significativamente el desarrollo académico en la UNA" (UNA, s. f., párr. 9).

Como modelo institucional, ha de ser conocido y plasmado en todas las actividades de la vida universitaria y de quienes se desempeñan en dicha institución. Por lo tanto, como orientador de nuestro accionar como académicos y académicas universitarias, debe verse reflejado en las dinámicas de aula, en las estrategias de enseñanza y aprendizaje que utilicemos y en cualquier actividad dentro de la vida universitaria, siempre en un marco de respeto y diversidad (UNA, 2008). Por tal razón, se han desarrollado sesiones y talleres de actualización sobre este Modelo pedagógico, dirigidos a los académicos y académicas que se desempeñan en las distintas unidades académicas.

En la línea señalada y como parte de la Política Institucional de Implementación del Modelo Pedagógico de la Universidad Nacional, surge la directriz de que la Universidad se aboque a distintos procesos para su implementación. Dicha instancia solicita, a las distintas facultades, centros y sedes, el cumplimiento de esta política, razón por la cual el Centro de Investigación y Docencia en Educación (CIDE), desde su Comisión de Gestión Académica y Curricular, ha instado a sus distintas Unidades Académicas a aproximarse a este proceso.

Desde la Comisión de Gestión Académica y Curricular se propusieron, como líneas de desarrollo académico en el Centro de Investigación y Docencia en Educación (CIDE), con apoyo de las Unidades Académicas, las siguientes áreas: integración curricular, incorporación de tecnologías e investigación.

Mediante el oficio CIDE-VD-281-2010 (CIDE, 2010), se hizo llegar a las Unidades Académicas y, posteriormente, a cada académico y académica, la directriz para su abordaje. En el caso de la División de Educación Básica, Unidad Académica adscrita al CIDE, se aclara que, para darle continuidad al trabajo que se ha venido realizando en los distintos niveles de las carreras y en busca de mayor coherencia para la formación del estudiantado, el área a la cual se abocarán, inicialmente, sus académicos y académicas, es a la de Integración curricular.

En razón de lo anterior, como académicos y académicas de la División de Educación Básica, nos hemos visto convocados al diseño y ejecución de diversas estrategias para promover la integración curricular en los distintos niveles del desarrollo de las carreras que se imparten. El presente artículo pretende sistematizar y compartir, con la comunidad universitaria, la experiencia que, en el sentido señalado, hemos vivido durante el primer ciclo del 2011, como académicos y académicas a cargo de los distintos cursos del primer nivel de diplomado de la carrera de Licenciatura en Pedagogía con Énfasis en Educación Preescolar.

\section{Desarrollo}

\section{Contextualización}

Iniciar un proceso de integración curricular, a criterio nuestro, requiere de una serie de acciones medulares, dentro de las que es posible destacar el conocimiento de las personas que 
conformarán el equipo de académicos y académicas a cargo de los distintos cursos que se impartirán en cada nivel, en el caso particular, en el primer nivel de diplomado de la carrera de Educación Preescolar. Por ello, se considera valioso recordar que la conformación de cualquier equipo de trabajo conlleva una serie de etapas complejas, las cuales requieren de tiempo para lograr la constitución como tal.

En ese sentido, Esquivel et al. (2004) señalan que los equipos están constituidos por una red de relaciones y vínculos entre personas, cuyo propósito es, entre otros, la búsqueda de objetivos comunes para compartir y generar, en ese intento, un sentido de pertenencia y apropiación del grupo que lo integra. Agregan los autores citados que una estrategia para promover la confianza, seguridad y estabilidad de los miembros de un equipo de trabajo, es el sentirse valorados, el que sus aportes, independientemente de su contenido, sean considerados importantes para el desarrollo del trabajo del grupo. En su criterio es esta una de las formas en que, poco a poco, el equipo se siente en la confianza de ir proponiendo alternativas de trabajo y definiendo las metas que desea conseguir.

Senge (2006, citado por Alfaro et al., 2010) señala que un equipo que se considere bien organizado ha de tener un aprendizaje en tres dimensiones:

1. La necesidad de pensar, agudamente, sobre los problemas complejos. Se aprende a pensar como colectividad.

2. La necesidad de una acción innovadora y coordinada. Se comprende que los actos de una persona complementan los actos de los demás.

3. El papel de los miembros del equipo en otros equipos. Un equipo que aprende alienta a otros a conformar una organización similar. (p. 171)

El análisis de esas tres dimensiones tiene en común el factor tiempo, pues como se ha dicho, el proceso de conformación de un equipo de trabajo no se logra en pocos días, sino que involucra una serie de aspectos no solo personales y profesionales, sino también, contextuales e institucionales; todos aspectos valiosos de considerar, cuando el objetivo primordial es generar experiencias de desarrollo que brinden oportunidades para una mejor entrega de la docencia.

Un equipo, por tanto, implica no solo la reunión de personas: es mucho más que eso, es un ejercicio de integración, de establecimiento de relaciones nuevas, de conocerse en un contexto diferente, de apoyarse, de sentirse en la libertad de preguntar, de equivocarse, de aprender juntos, de abocarse a nuevos trabajos y nuevos retos; es, como puede verse, una nueva experiencia que debe llevarse al ritmo de las dinámicas interactivas que a su interior se generen. Es un cúmulo de procesos que no pueden precipitarse, ni obligarse, para los cuales ha de existir el tiempo necesario para cumplir con las distintas etapas propias de cualquier grupo de trabajo en busca de metas comunes. Como señalan Alfaro et al. (2010, p. 173): "El equipo emerge de la interacción de las personas, lo que permite a todos sus miembros evolucionar mediante una dinámica que ningún otro equipo tendrá, es decir, cada equipo es único e irrepetible (...)".

Así como un equipo implica el establecimiento de relaciones y dinámicas interactivas que se generan a su interior, de igual manera el salón de clase en el cual interaccionan docentes y estudiantes, también, tiene esas dinámicas. En una investigación reciente (Pereira, 2010a), 
la autora señala que quienes mayor relación tienen con el estudiantado, son los docentes y las docentes y que en el salón de clase, donde se establecen esas dinámicas interactivas, juegan un papel preponderante docentes y alumnos, pero también lo hacen los contenidos, las estrategias de aprendizaje que se desarrollan y, por supuesto, el clima de aula que se genere como resultado de la interacción de dichos aspectos, dado que el contexto educativo no se encuentra exento de procesos comunicativos e interactivos. Por lo dicho, compartimos la idea de que los equipos académicos han de estar en constante auto-reflexión sobre la práctica profesional docente, pues sus acciones tienen repercusiones directas sobre el trabajo de aula y sus estudiantes, de ahí que todos los aspectos del currículo poseen importancia en el acto educativo y, con más razón, han de ser considerados cuando se hacen esfuerzos por diseñar y aplicar estrategias integradas en los procesos educativos.

Por otra parte, Águila, citado por Mora (s. f.) manifiesta que la integración curricular no significa poner juntos los contenidos de diversas materias, sino más bien significa organizar los contenidos temáticos del currículo en actividades que permitan la globalización de los saberes. En ese sentido, agrega el autor que:

(...) se pretende superar la separación por asignaturas de las áreas del conocimiento, la fragmentación de los aprendizajes, de manera que el aprendizaje sea funcional. Es decir, que el alumno lo vea funcionando en una situación o problema real y construya las estrategias que le permitan establecer nuevas relaciones significativas entre contenidos diversos siendo capaz de realizar aprendizajes significativos por sí mismo, en una amplia gama de situaciones y circunstancias. (párr. 4)

En concordancia con lo expuesto, Gaff (1989), citado por Quintana (1998) y ellos citados en Mora (s. f.), plantea que existen tres argumentos para promover la búsqueda de la integración: el primero señala que las ideas en cualquier campo de estudio son siempre enriquecidas por los conocimientos, teorías y conceptos de otros campos. El segundo plantea que es mejor para el aprendizaje del estudiantado que se presente el conocimiento organizado en unidades completas y no aisladas, lo que presupone, entonces, que es mejor presentar el conocimiento integrado y no de manera aislada. Y el tercer grupo de argumentos para la integración señala que, aunque el aprendizaje se visualiza como una actividad individual, lo cierto es que eso no significa que debe hacerse en solitario, ya que es más efectivo cuando se concibe el salón de clase como una comunidad de aprendizaje en la cual todos dan sus aportes y promueven, de esa manera, el aprendizaje propio y el de los compañeros y compañeras. Por ello, añade que los cursos que se diseñan o buscan actividades integradas suelen interesar más al estudiantado.

Por otra parte, Beane (2005) plantea que la teoría sobre la integración curricular se puede ubicar en los siguientes campos: la integración de la experiencia, la integración social, la integración de los conocimientos y la integración como diseño curricular. De ahí la importancia de clarificar en qué ámbitos se desea llevar a cabo el ejercicio de integración curricular y valorar, no solo las estrategias a seguir, sino también los alcances de las mismas.

Reiteradamente, en el campo pedagógico, se señala la necesidad de evitar la fragmentación del conocimiento, así como evitar el distanciamiento entre la institución educativa y la cotidianeidad. Por tanto, es viable suponer que la mejor manera de lograr la construcción de conocimientos pertinentes y significativos pueda serlo mediante esfuerzos por realizar actividades que permitan 
la integración curricular en un inicio y lograr desarrollar estrategias que admitan el diseño de un currículo integrado como meta futura.

Apostar por la búsqueda de alternativas para la integración curricular es una tarea posible dentro de los equipos de académicos y académicas que deseen abocarse a ello (Sánchez, s. f.). Y en ese sentido, creemos también posible que para el estudiantado es mucho más rica la experiencia del aprendizaje cuando puede analizar problemáticas de manera integrada.

Agrega Sánchez (s. f.) que promover actividades o acciones de integración curricular conlleva la idea básica de entender y profundizar en temas o problemas desde una visión más holística, desde donde se contempla la posibilidad de considerar diferentes ángulos para la atención de un problema en particular: buscar un objetivo; aprender un concepto; desarrollar un proceso investigativo o comprender una visión disciplinar específica, entre otros, y con la consideración de los aportes de diferentes disciplinas. Esta visión integral implica usar, de manera armónica y funcional, los aportes de distintas disciplinas para un propósito de aprendizaje específico.

Iran-Nejad, McKeachie y Berliner (1990), citados por Beane (2005), señalan que “(...) cuanto más significativo es un suceso, cuanto más se procese en profundidad y detalle, cuanto más contextualizado esté, y cuanto más enraizado en los conocimientos culturales, históricos, meta cognitivos y personales, más fácilmente se comprende, se aprende y se recuerda (...)” (p. 25).

Ahora bien, podría decirse que este trabajo de integración curricular, es un trabajo interdisciplinario. Al respecto, Piaget (1979), citado por Torres (2006), plantea que la interdisciplinariedad es el "(...) segundo nivel de asociación entre disciplinas, donde la cooperación entre varias disciplinas lleva a interacciones reales; es decir, hay una verdadera reciprocidad en los intercambios y, por consiguiente, enriquecimientos mutuos" (p. 72).

Sumado a lo anterior, Torres (2006) destaca que el currículo se puede organizar no solamente en asignaturas, como siempre se ha hecho, sino puede superar los límites de las disciplinas, con el propósito de que los estudiantes se vean obligados a manejar conceptos, teorías, procedimientos y destrezas de diversas disciplinas, para dar solución a las situaciones o problemas planteados. Precisamente, uno de los objetivos del trabajo que desarrollaron las estudiantes consistió en que pudieran obtener los aportes de los diferentes cursos del nivel para hacer una investigación de una situación real relacionada con la educación preescolar.

Queda en evidencia, entonces, que el aspecto práctico y las vivencias diarias se convierten en una oportunidad para realizar procesos interdisciplinarios. Al respecto, Torres (2006) rescata lo siguiente:

La interdisciplinariedad es un objetivo nunca alcanzado por completo y de ahí que deba ser permanentemente buscado. No es sólo un planteamiento teórico, es ante todo una práctica. Su perfectibilidad se lleva a cabo en la práctica, en la medida en que se hacen experiencias reales de trabajo en equipo, se ejercitan sus posibilidades, problemas y limitaciones. Es una condición necesaria para la investigación y la creación de modelos más explicativos de esta realidad tan compleja y difícil de abordar.

(...) Es claro, que desde donde se ve con mayor apremio la necesidad de interdisciplinariedad es desde las situaciones prácticas. Los problemas urgentes e inevitables de la vida práctica precisan soluciones que no pueden demorarse $\mathrm{y}$, por consiguiente es más fácil solicitar la colaboración de personas de todas las especialidades que tengan algo que decir sobre esa situación a resolver (...). (p. 69) 
De este modo se puede señalar que aspectos como la cooperación, la integración, la interdisciplinariedad y el enriquecimiento mutuo fueron característicos de este trabajo desarrollado en conjunto en el primer nivel de diplomado de la carrera de Educación Preescolar, para lograr un objetivo en común y un mayor conocimiento de la realidad, tomando como base diferentes aportes de los cursos de dicho nivel y del profesorado a su cargo.

En concordancia con lo expuesto en las páginas precedentes, como equipo de académicos y académicas, desde el momento de recibir la instrucción de abocarnos a la búsqueda de estrategias de integración curricular, en las primeras reuniones de trabajo, establecimos algunos principios, que a nuestro criterio, eran medulares para la consecución de metas en común, entre los que se pueden señalar:

1. Como equipo de nivel optamos por una visión amplia de integración en términos de un proceso de construcción de objetivos comunes, para logros tanto de estudiantes, como de académicos y académicas. Para alcanzar un trabajo integrado consideramos importante la disposición de las académicas y los académicos del nivel para colaborar y flexibilizar aquellos aspectos de los cursos contemplados en el objetivo común, razón por la cual, según nuestro criterio, no pueden existir lineamientos unidireccionales para los procesos de integración que se promueven en los equipos del nivel. El respeto a las decisiones de los diferentes grupos de trabajo y las opciones de integración que se asuman en cada nivel, deben verse como parte de ese proceso que implica la integración del equipo.

Para realizar el trabajo por nivel, se comparte el criterio de que trabajar de forma integrada implica no solamente trabajar en función de un objetivo programático, sino también, en función de la formación de las estudiantes (todas eran mujeres) en muchos otros aspectos referidos a su buen desenvolvimiento (puntualidad, respeto, no hacer trabajos de otros cursos durante la clase, llegadas tardías por imprimir un trabajo, uso correcto de las normas de la American Psychological Association (APA) para la presentación de trabajos, entre otros).

2. Reconocer que la integración curricular se ha de concebir como un proceso paulatino, por lo que va a depender mucho de la estabilidad de los académicos y académicos en el nivel. Se comparte la idea de que a medida que los equipos se consolidan, en esa misma medida, se van fortaleciendo los procesos de integración.

4. Valorar el respeto por la individualidad profesional y contenidos del curso. No se puede obligar a nadie a que cambie sus estrategias de trabajo que van de la mano con su manera de ver el aprendizaje y la docencia. Pero si se puede negociar, en qué aspectos y cómo sería posible realizar esfuerzos de integración.

5. Recurrir por parte del equipo de académicos y académicas de nivel a estrategias comunicativas más eficientes para complementar las reuniones presenciales, a saber, teléfono, correo electrónico, entre otras opciones viables.

6. Propiciar el abordaje de las actividades y trabajos (en nuestro caso, específicamente en torno al trabajo final de investigación de los cursos) de las estudiantes de forma coordinada e integrada, apoyando desde todos los cursos el fortalecimiento del marco teórico, diseño de estrategias y otros aspectos medulares, para realizar, por parte de ellas, una actividad curricular integrada por nivel.

7. Abordar unificadamente las normas de (APA) para la presentación de trabajos científicos vigentes en el CIDE, con el propósito de que desde todos los cursos se revise y apoye el trabajo estudiantil. 
8. Atender a las estudiantes que requieren apoyo o asesorías, y vigilar el avance a nivel académico y personal, del grupo de estudiantes desde todos los cursos.

\section{Experiencia de integración curricular}

Una vez explicitados los principios que enmarcaron el trabajo a realizar por parte del equipo de académicos y académicas, es pertinente, a continuación, compartir lo que ha sido el desarrollo de la experiencia Se inicia por una presentación de los distintos cursos que han sido objeto del ejercicio de diseñar e implementar estrategias de integración curricular, a saber: Introducción a la investigación, Desarrollo humano y Educación, Expresión Plástica en la Educación Preescolar, Música en la Educación Preescolar y Evolución de la Educación Preescolar. Para ello, retomaremos en primer lugar los principios del Modelo pedagógico (UNA, 2008) que guiaron el trabajo a desarrollar dentro del primer nivel de diplomado, con el propósito de contextualizar la experiencia desarrollada. Los mismos constituyen todo el bloque de cursos de dicho nivel.

Con el fin antes descrito, para contextualizar la experiencia desarrollada, se retoman a continuación, en primer lugar, los principios del Modelo pedagógico de la UNA, a saber:

- Respeto a la diversidad en todas sus expresiones.

- Respeto y compromiso con la igualdad de oportunidades y con la construcción de una sociedad más justa y equitativa.

- Formación de profesionales solidarios y comprometidos con el bienestar social.

- Flexibilidad para conceptuar el aprendizaje como proceso sociocultural, histórico, dinámico y transformable, posible y que puede construirse de muchas maneras.

- Interacción en los procesos formativos donde los conocimientos sean discutidos y enriquecidos permanentemente.

- Formación de un espíritu investigador en los futuros profesionales.

- Creatividad que permita la innovación, así como la utilización de medios, estrategias y recursos de enseñanza en los procesos de mediación pedagógica.

- Disposición para determinar los principios lógicos subyacentes en cada disciplina, que permitan una formación profesional de calidad.

- Evaluación como proceso integral, concertado, permanente, contextualizado y propositivo.

- Mejoramiento continuo en la formación integral de los estudiantes y los procesos de gestión académica-administrativa y paraacadémica.

- Visión prospectiva que permite la planificación estratégica para el logro de objetivos a mediano y largo plazo. (UNA, 2008, p. 5)

Por consiguiente, una de las primeras acciones realizadas por el equipo fue identificar, para el diseño de la integración curricular, cuáles de esos principios podrían ser asumidos como ejes transversales en cada uno de los cursos que tenían a cargo los académicos y las académicas del primer nivel de diplomado que se estaban conformando como equipo de trabajo. 
Así, por ejemplo, en el curso de Introducción a la investigación se identificaron como prioritarios los principios de mejoramiento continuo en la formación integral de las estudiantes, el de interacción en los procesos formativos y el de formación de un espíritu investigador.

Igualmente, en el curso Desarrollo humano y Educación, se trabajó con los principios de mejoramiento continuo en la formación integral de las estudiantes, la formación de un espíritu investigador, el respeto a la diversidad en todas sus expresiones, la flexibilidad para conceptuar el aprendizaje como proceso sociocultural, histórico, dinámico y transformable y la evaluación como proceso integral.

En el curso Evolución de la Educación Preescolar, se retomaron los principios de flexibilidad para conceptuar el aprendizaje como proceso sociocultural, histórico, dinámico y transformable, posible y que puede construirse de muchas maneras; así como los principios de interacción en los procesos formativos donde los conocimientos sean discutidos y enriquecidos permanentemente, $\mathrm{y}$ el de formación de profesionales solidarios y comprometidos con el bienestar social.

Por otra parte, en el curso de Expresión plástica en la Educación Preescolar, se tomaron en cuenta los principios de respeto a la diversidad en todas sus expresiones, el de interacción en los procesos formativos donde los conocimientos sean discutidos y enriquecidos permanentemente, así como el de creatividad que permita la innovación.

En el curso de Música en la Educación Preescolar, se fomentó el principio de interacción en los procesos formativos donde los conocimientos sean discutidos y enriquecidos permanentemente, el principio de formación de un espíritu investigador en los futuros profesionales, y el principio de creatividad que permita la innovación, así como la utilización de medios, estrategias y recursos de enseñanza en los procesos de mediación pedagógica.

Así, todos los principios señalados fueron retomados por el equipo de académicos y académicas con el propósito de que las estudiantes pudieran ponerlos en práctica en las diferentes experiencias y dinámicas que desarrollaron en el aula universitaria y, al mismo tiempo, que pudieran propiciarlos en el momento de trabajar con los niños y niñas de edad preescolar.

De acuerdo con lo dicho anteriormente y como parte de las estrategias de integración curricular y su referente con el Modelo pedagógico (UNA, 2008), cada uno de los cursos llevó a la práctica acciones concretas con el propósito de hacer efectivos los distintos principios del Modelo que fueron identificados y asumidos como ejes transversales para el desarrollo de la experiencia de integración curricular. A continuación, se describen las acciones seguidas en cada uno de los cursos, como ejemplo de lo que fue esta experiencia de incorporar aspectos del Modelo pedagógico como ejes medulares en cada curso.

\section{Curso: Introducción a la investigación}

En el curso Introducción a la investigación se enfatizó en los procesos formativos que responden al principio del mejoramiento continuo en la formación integral de las estudiantes. Se concretó dicho aspecto en la elaboración de un portafolio que valoró el avance del estudiante en cuanto al manejo de contenidos y responsabilidades asignadas como parte del curso, el cual finalizó con un ejercicio de autoevaluación. 
El principio de interacción en los procesos formativos y el de formación de un espíritu investigador se constituyeron, para el curso de investigación, en ejes transversales del quehacer en dicho curso, así como para los otros cursos del nivel. Por lo que han estado presentes no solo en los procesos observacionales que las estudiantes realizaron en instituciones educativas, sino también, en el diseño y delimitación de un tema de investigación en el ámbito educativo.

El programa de este curso incorporó los aspectos que cada uno de los académicos y las académicas de nivel estipularon que se requería encontrar en el informe final y para lo cual brindaron aportes a los estudiantes, por tanto, este curso, como curso eje o conductor de la investigación que realizaron las estudiantes, pautó:

La integración del trabajo final para el cumplimiento de la actividad curricular del nivel, se hizo efectiva mediante dos acciones concretas:

- El trabajo de investigación en el campo pedagógico, fortalecido desde los otros cursos del nivel.

- La estructura el trabajo escrito (formato, lineamientos y pautas de un informe de investigación).

\section{Curso: Desarrollo humano y Educación}

En el curso de Desarrollo humano y Educación se contemplaron diferentes espacios evaluativos en el aula, que les permitieron a las estudiantes valorar el crecimiento personal de manera que experimentaran un mejoramiento continuo en su formación integral.

El principiorelacionadocon la formación de un espíritu investigador se trabajófundamentalmente a partir de las visitas y observaciones que realizaron las estudiantes a diversas instituciones. Dichas observaciones exigían una continua reflexión de los procesos de desarrollo presentes en las niñas y los niños preescolares y una identificación de aspectos a investigar para mejorar la práctica educativa a partir de la atención integral del desarrollo humano. En ese sentido, la temática de investigación que ellas iban seleccionando debía comprenderse teniendo en cuenta una perspectiva de desarrollo humano integral, en la que se contemple, al menos, los siguientes aspectos:

- Características de las áreas del desarrollo humano, según la edad y nivel de las niñas y los niños.

- Promoción de procesos integrales de desarrollo humano, según la aplicación de distintas estrategias de expresión plástica y musical.

- Integralidad de los procesos de desarrollo.

- Factores que influyen en el desarrollo humano.

Con el propósito de realizar un proceso de aprendizaje significativo, al abordar el desarrollo humano se incorporaron experiencias vividas por las estudiantes, valorando siempre el respeto a la diversidad en todas sus expresiones. Se comprendió el porqué de las diferencias individuales y cómo atenderlas de manera inclusiva. Lo anterior posibilitó, también, la conceptualización del aprendizaje como proceso sociocultural, histórico, dinámico y transformable.

Finalmente, la evaluación realizada a lo largo del curso se hizo como un proceso integral, teniendo en cuenta los objetivos y perfiles planteados en el plan de estudios. Asimismo, la evaluación de los aprendizajes consideró tanto el nivel cognitivo real, como el progreso y el

72 Ana Lucía Chaves Álvarez, Dora Hernández Vargas, Jorge León Sánchez, Zulay Pereira Pérez y Marie Claire Vargas Dengo 
crecimiento a lo largo del curso e hizo posible que, en el trabajo de investigación, los conocimientos construidos se vieran plasmados en propuestas acordes con las teorías de desarrollo estudiadas.

\section{Curso: Expresión plástica en la Educación Preescolar}

El curso Expresión plástica en la Educación Preescolar propició el respeto a la diversidad en todas sus expresiones, en las diferentes experiencias que se desarrollaron en el aula universitaria, así como en las diferentes asignaciones que tuvieron que presentar las estudiantes. Se está hablando específicamente de lo siguiente:

a) Lecturas: análisis, comentarios y reflexiones sobre diferentes textos relacionados con la temática del curso.

b) Propuesta de creación de un área de artes plásticas

c) Carpeta: Técnicas gráfico plásticas y etapas evolutivas de la expresión gráfica del niño.

d) Trabajo integrado de nivel.

La interacción en los procesos formativos, donde los conocimientos se discutan y enriquezcan permanentemente, fue un principio que se concretó en la dinámica del aula universitaria con las opiniones y aportes de las estudiantes, relacionados con las temáticas del curso. Asimismo, a lo largo de este, se promovió el análisis y la relación entre lo teórico y las experiencias vividas en los diferentes centros infantiles que visitaban.

Por la naturaleza del curso, el principio de creatividad que permita la innovación, fue trascendental en el proceso formativo, ya que lo que se pretende es que el estudiantado explote al máximo su potencial creativo, para que a la vez puedan propiciar esto con los niños y niñas de edad preescolar.

Ahora bien, específicamente en cuanto al trabajo integrado de nivel, desde este curso se estableció que, de acuerdo con la investigación que cada subgrupo fuera a desarrollar, en el informe final de investigación se podían contemplar algunas temáticas específicas planteadas por la académica, o algunas otras que emergieran de la iniciativa de las estudiantes, siempre y cuando estuvieran relacionadas con el curso. Las temáticas propuestas fueron las siguientes:

- Creatividad y su relación con la expresión plástica del niño preescolar.

- Etapas evolutivas de la expresión gráfica del niño.

- Papel del maestro en la orientación y estimulación de la expresión plástica en el niño preescolar.

- Importancia de las técnicas gráfico plásticas en el desarrollo del niño preescolar.

- Importancia del arte en el desarrollo y aprendizaje del niño preescolar.

- Integración de las artes plásticas con las otras áreas del currículo en preescolar.

- Evaluación de la expresión gráfico plástica del niño.

- Técnicas gráfico plásticas desarrolladas en clase. 
Además de lo anterior, otro aporte del curso al trabajo integrado consistió en una experiencia de aplicación de técnicas gráfico plásticas. Tal como se explicó anteriormente, las estudiantes desarrollaron en clase diferentes técnicas gráfico plásticas, las cuales presentaron en una carpeta. Ellas seleccionaron cuatro de las dieciséis técnicas que se hicieron en clase y las aplicaron con los niños y niñas, en un aula preescolar. Este aporte fue tomado en cuenta por las estudiantes e incorporado en el apartado de metodología del trabajo final de investigación.

\section{Curso: Música en la Educación Preescolar}

En este curso se concibió el diálogo como una fuente de construcción y reconstrucción del conocimiento, de manera que el principio de interacción en los procesos formativos que propone el Modelo pedagógico de la UNA (2008) fue desarrollado mediante espacios de interacción en talleres y equipos de trabajo. La formación de un espíritu investigador en los futuros profesionales, planteado por este Modelo, se concretó en la construcción del trabajo final de investigación, como hemos señalado, de manera integrada. La lógica en la cual se insertó la investigación en este curso en relación con los demás del nivel se explica de la siguiente manera: el curso de Investigación sería el que llevaría la batuta en torno al proceso mismo de la investigación, las estrategias metodológicas a desarrollar y del formato de presentación del informe de investigación como tal. El curso de Evolución de la Educación Preescolar aportaría el análisis del contexto en el cual las estudiantes llevarían a cabo la investigación; el curso de Expresión plástica en la Educación Preescolar, así como el curso de Música en la Educación Preescolar, aportarían herramientas teórico conceptuales para que las estudiantes propusieran o implementaran estrategias pedagógicas a partir del uso de la música y la plástica en el jardín infantil; y el curso de Desarrollo humano y Educación permitiría a las estudiantes formular propuestas acordes con el nivel, necesidades y características de los niños y niñas.

En este sentido, la música fue abordada en el curso no solo como un área de conocimiento que enriquece la vida en el aula preescolar, sino como una herramienta para la construcción de aprendizajes más significativos y deleitosos. Los procesos de investigación se concibieron como espacios para construir caminos y propuestas hacia opciones pedagógicas que, tomando en cuenta la música, permitieron a las estudiantes y a los niños con quienes trabajaron, potenciar relaciones y espacios de aprendizaje.

La creatividad que permita la innovación, el último de los tres principios del Modelo desarrollados en el curso, fue promovida con la misma intencionalidad de los procesos de investigación, de aportar a la capacidad de las estudiantes para propiciar espacios adecuados de aprendizaje. Sin embargo, esta creatividad se promovió en dos vertientes: una de carácter más personal, donde se buscaba el desarrollo de la sensibilidad estético creadora de la estudiante y otra de carácter más docente, donde se buscaba el desarrollo de la capacidad para enfrentar de forma creativa el ambiente pedagógico. De manera que desde la primera vertiente, las estudiantes solo debían preocuparse por disfrutar y apreciar la música y el arte para ellas mismas, y desde la segunda vertiente, debían ligarlo con lo pedagógico. Ambas dimensiones están sumamente conectadas entre sí, pero en el curso se estableció una diferenciación con el fin de asegurarnos un abordaje más integral. 


\section{Curso: Evolución de la Educación Preescolar}

El curso Evolución de la Educación Preescolar incursiona en el desarrollo histórico de la educación preescolar para comprender los fundamentos filosóficos y pedagógicos, así como los cambios relevantes en la educación de la población infantil en el transcurso del tiempo.

Con el propósito de retomar el principio de conceptuar el aprendizaje como un proceso socio-histórico y cultural posible de construir de distintas maneras y de transformar en el tiempo y el espacio, se enfatizó la funcionalidad de los aprendizajes a partir de la relación entre la teoría y las vivencias prácticas del estudiantado, procurando una constante transferencia de los aprendizajes al contexto de aula preescolar o programa de atención infantil, así como de los aprendizajes en dichos contextos al aula universitaria. Se propició la interacción en los procesos formativos por medio de la discusión de conocimientos dada al compartir e intercambiar puntos de vista sobre distintas situaciones de aprendizaje, tanto desde la teoría como desde la vivencia personal. En este sentido, es relevante la asistencia a los distintos centros educativos y programas infantiles, como también lo es para la formación de profesionales solidarios y comprometidos con el bienestar social, capaces de analizar la realidad de la niñez costarricense en diferentes ámbitos.

Contextualizaron los temas de escogencia grupal en programas de atención y educación infantil, tales como: Jardines (públicos, privados), guarderías, CEN CINAI (urbano, rural), hogares comunitarios y albergues. Esto con el fin de que las estudiantes conozcan y se familiaricen con la realidad cotidiana en la que participarán como futuras docentes.

Los espacios seleccionados corresponden a los lugares asignados para las visitas del curso, correspondientes a la intervención pedagógica en las horas de práctica. En este sentido y según lo especifica el eje práctico pedagógico de la carrera de Pedagogía con énfasis en Educación Preescolar (División de Educación Básica. CIDE, 2008) la Intervención Pedagógica (IP):

Implica un proceso de inserción progresiva del estudiante en la realidad del aula, contextualizada dentro de una dinámica socio-cultural e histórica lo que permite un encuentro del estudiante con la realidad, a partir de la cual se construyen perspectivas teóricas propias, susceptibles a la confrontación y a la reconstrucción constante. (...) Es decir se busca que el estudiante parta de experiencias prácticas, como un medio para investigar, analizar y generar procesos tendientes a lograr cambios en la realidad educativa (p. 40)

\section{Estrategias definidas por el equipo académico para la integración}

Haciendo un recuento de las experiencias vividas como equipo de académicos y académicas en la búsqueda por construir espacios de integración curricular, es posible identificar algunas de las estrategias que se siguieron en función del objetivo común:

- El equipo de académicas y académicos de este nivel inició con la discusión de consideraciones preliminares sobre los lineamientos dados acerca de la integración curricular por parte de la administración. 
- Se identificaron y compartieron las temáticas de los cursos, los principios del Modelo pedagógico (UNA, 2008) que más compatibilidad tenían con el trabajo a desarrollar en cada curso, así como las vías mediante las cuales parecía pertinente llevarlos a la práctica cotidiana en el aula.

- Se acordó una propuesta por etapas, de modo que, inicialmente, se plantearían algunos principios básicos para el trabajo; se definirían algunas estrategias posibles de trabajo; se buscaría consenso en cuanto a la actividad curricular por desarrollar, entre otros aspectos

- Se acordó definir un cronograma para calendarizar los pasos y dar seguimiento en cada curso.

- Se acordó trabajar minuciosamente en la revisión de la redacción, la ortografía y las referencias de los trabajos, en acato a las normas del APA adaptadas para el CIDE (Zúñiga y López, 2003) y tomando medidas en la revisión y evaluación de los trabajos asignados.

- Se programó la elaboración de una investigación integrada por parte de las estudiantes, mediante la cual se buscaba la integración de los saberes y competencias de los distintos cursos del nivel. Para ello, cada académico y académica en el programa de su propio curso, indicó los aspectos y criterios de evaluación que asignó a dicho trabajo. De igual manera, estos fueron incorporados en los criterios que para el informe final de investigación se establecieron en este programa en un documento común que se entregó a las estudiantes.

- Se programó una presentación final de los trabajos de las estudiantes ante el académico y académicas del nivel y sus compañeras de curso, con una entrega previa del trabajo escrito en formato digital.

- En cada curso se explicó a las estudiantes el trabajo por realizar.

- Desde el inicio del ciclo lectivo, se conformaron 7 grupos de trabajo por parte de las estudiantes.

- Las estudiantes definieron un tema de investigación para realimentarlo con los aportes de todos los cursos del nivel.

- Las estudiantes retomaron, de cada curso, contenidos temáticos pertinentes a su tema para fortalecer la introducción y el marco teórico.

- Las estudiantes entregaron a cada académico y académica un informe del trabajo final en versión digital.

- Las estudiantes realizaron presentaciones orales de los trabajos ante el equipo de académicos y académicas del nivel y sus compañeras de curso.

- Se propuso, de común acuerdo, que cada académico y académica calificara el trabajo en cada curso. En cada uno de los programas respectivos se incluyó un rubro sobre el trabajo final integrado, tanto en los objetivos como en la evaluación. Además, las estudiantes recibieron una única nota para dicho trabajo, la cual fue el resultado del promedio de calificaciones que las académicas y el académico del primer nivel de diplomado le asignaron al informe final de investigación.

- En cuanto al punto anterior, vale resaltar que mediante comunicación por correo electrónico el equipo de académicos y académicas compartió la revisión de aquellos trabajos que por alguna razón generaban dudas o cuestionamientos, de modo que esta estrategia facilitó mucho la búsqueda de consenso y el aportar una nota más objetiva. Fue muy interesante poder compartir opiniones y apreciaciones que en distinto momento le surgieron a algún miembro del equipo y que mediante el recurso en línea fue muy saludable compartirlas y analizarlas en la búsqueda de la mejor alternativa de solución. 
- También, fue muy significativo el poder compartir material de lectura y apoyo, que en diferentes momentos llegó a nuestras manos y hacer partícipe de ello al resto de compañeros y compañeras de nivel.

\section{Actividades de seguimiento}

El grupo de académicos y académicas, una vez definidas las etapas para alcanzar la integración curricular definida, se abocó a la realización de acciones como las siguientes:

- Nombramiento de una coordinadora del grupo, a fin de poder organizarnos de una mejor manera.

- Consignar en crónicas los acuerdos del grupo de académicos del primer nivel de diplomado, las cuales se hacían llegar vía correo electrónico a cada uno de los miembros del grupo para su revisión y valoración de acuerdos. De modo que fueron sistemáticamente compartidas.

- Reuniones presenciales.

- Comunicaciones constantes vía electrónica para atender diferentes aspectos que se fueran presentando a lo largo del ciclo lectivo.

- Seguimiento de las estudiantes, en cuanto a su desempeño personal y académico. Se compartió vía correo electrónico el análisis de distintas situaciones vividas por las estudiantes y en algunos casos se buscaron alternativas de solución con apoyo de la Dirección de la Unidad Académica.

- En cada curso se proporcionaron las indicaciones concernientes a la investigación integrada que se realizaría y a los aspectos relativos a cada curso.

- Sesiones individuales con los grupos de trabajo en algunos de los cursos.

- Apertura de espacios durante las sesiones de clase, para consultas de las estudiantes, orientación y aportes para el enriquecimiento de la investigación.

- Cada académico y académica revisó y brindó sugerencias y aportes a los distintos trabajos de las estudiantes, de modo que cada grupo de estudiantes recibió cinco devoluciones con sus respectivas sugerencias.

- Valoración individual por parte de cada docente de las presentaciones orales sobre los resultados de la investigación integrada.

- Ponderación de las calificaciones de cada académica y académico para asignar una sola calificación.

- Devolución de los trabajos con sus respectivas calificaciones a las estudiantes.

- Aplicación de un instrumento mediante el cual las estudiantes del primer nivel de diplomado valoraron la experiencia de integración realizada.

\section{Valoración estudiantil acerca de la experiencia de integración curricular realizada en el primer nivel de diplomado}

Cabe mencionar que, a las 32 estudiantes que asistieron a los distintos cursos del primer nivel de diplomado de la carrera de Educación Preescolar, se les aplicó un cuestionario, al finalizar el ciclo lectivo, para conocer su opinión sobre el trabajo de integración curricular realizado en este nivel de carrera. El instrumento se diseñó para indagar básicamente lo siguiente: 


\section{URL: http://www.una.ac.cr/educare}

a. Los aspectos positivos del trabajo de integración curricular para su formación profesional.

b. Lo más significativo de la experiencia de realizar el trabajo integrado.

c. Los aspectos que les representaron mayor dificultad en la realización del trabajo.

Una vez que las estudiantes completaron el instrumento, se recolectaron los cuestionarios para transcribir la información obtenida. Los datos se organizaron en función de las tres interrogantes planteadas, a partir de las cuales se definieron tres categorías generales. Con la información obtenida y en concordancia con las categorías definidas, se establecieron categorías emergentes y se acotaron aspectos propiamente derivados de los datos obtenidos. A continuación se presentan los componentes básicos de las matrices que se utilizaron con el fin de vaciar y organizar la información para el posterior análisis e interpretación.

Tabla 1

Aportes que la actividad de integración curricular dio a la formación profesional, según opinión de las estudiantes participantes en la experiencia

\begin{tabular}{|c|c|}
\hline \multicolumn{2}{|c|}{ 1. Categoría: Aportes para la formación profesional } \\
\hline Categorías emergentes & Aspectos \\
\hline Aprendizaje y crecimiento en el ámbito académico & $\begin{array}{l}\text { Conocimientos e información de utilidad a futuro } \\
\text { Aprendizajes para: } \\
\text { - Investigar } \\
\text { - Elaborar otros trabajos } \\
\text { - Realizar el trabajo final de graduación } \\
\text { Aprendizajes sobre: } \\
\text { - El tema que investigaron } \\
\text { - La aplicación de lo aprendido en el aula universitaria } \\
\text { Adquisición de: } \\
\text { - Experiencia y confianza, para trabajar con niños y niñas } \\
\text { de edad preescolar. }\end{array}$ \\
\hline Aprendizaje y crecimientoen el plano personal & $\begin{array}{l}\text { Vivencias personales } \\
\text { Importancia de: } \\
\text { - Trabajar y relacionarse con muchas personas diferentes } \\
\text { - Escuchar otras opiniones } \\
\text { - Respetar distintos criterios } \\
\text { - Interactuar con las compañeras } \\
\text { - Solucionar problemas } \\
\text { - Desarrollar el sentido de responsabilidad y de } \\
\text { compromiso en el cumplimiento de tareas. }\end{array}$ \\
\hline
\end{tabular}

A partir de los datos obtenidos en la primera categoría, tal como se aprecia en la Tabla 1, cabe señalar que la totalidad de las estudiantes (32) valoraron positivamente la experiencia del trabajo realizado en términos de aprendizaje y crecimiento, tanto en el plano académico como en 
el personal. En el ámbito académico las participantes manifestaron que el trabajo integrado les permitió su crecimiento y se refirieron al aprendizaje de conocimientos e información de utilidad a futuro; destacaron los aprendizajes sobre investigación y cómo este trabajo las preparó para realizar otros trabajos más adelante en la carrera, inclusive algunas lo proyectaron a futuro aludiendo a la realización de su trabajo final de graduación; otras se refirieron a cuánto aprendieron sobre el tema que investigaron y cómo la puesta en práctica les permitió aplicar lo aprendido en el aula universitaria, además de adquirir experiencia y confianza, para trabajar con niños y niñas de edad preescolar.

En el plano personal, las estudiantes valoraron los aprendizajes en términos de vivencias y experiencias propias, en tanto que consideran que aprendieron 'muchas cosas' como por ejemplo que durante la carrera van a tener que trabajar y relacionarse con muchas personas diferentes, por lo que reconocen la importancia de escuchar otras opiniones, respetar criterios, interactuar con las compañeras, solucionar problemas, desarrollar el sentido de responsabilidad y compromiso en el cumplimiento de tareas, aspectos que coinciden plenamente con lo planteado por Pereira (2010b) cuando indica que las estudiantes valoran positivamente las estrategias de aula que les permiten desarrollar aspectos personales y profesionales como parte de sus aprendizajes.

En términos generales, por parte de las estudiantes, se valora positivamente el esfuerzo, empeño y dedicación que les implicó realizar el trabajo integrado, así como el tiempo invertido. Algunas expresaron sentir una base firme para su futuro profesional, así como que lograron desarrollar un espíritu investigador que les ayudará a orientarse como futuras docentes.

Tabla 2

Opiniones estudiantiles acerca de la significatividad de la experiencia mediante la actividad de integración curricular

\begin{tabular}{|l|l|}
\hline \multicolumn{2}{|c|}{ 2. Categoría: Significatividad de la experiencia } \\
\hline Categorías emergentes & \multicolumn{1}{c|}{ Aspectos } \\
\hline Relaciones interpersonales & $\begin{array}{l}\text { - Interacción con las compañeras de clase } \\
\text { - Interacción con las niñas y los niños de edad preescolar }\end{array}$ \\
\hline $\begin{array}{l}\text { Inserción en contextos educativos } \\
\text { y espacios de atención infantil }\end{array}$ & $\begin{array}{l}\text { - Temor inicial de trabajar con } \\
\text { - Visitas a centros educativos y programas de atención infantil }\end{array}$ \\
\hline $\begin{array}{l}\text { Aprendizajes sobre la elaboración del } \\
\text { trabajo final }\end{array}$ & $\begin{array}{l}\text { - Importancia de la formalidad } \\
\text { - Saber cómo elaborar un trabajo de investigación } \\
\text { Aprendizajes sobre la experiencia } \\
\text { del trabajo de integración }\end{array}$ \\
\hline
\end{tabular}


Con respecto a lo que les resultó más significativo de la experiencia, la Tabla 2 muestra que las estudiantes participantes destacaron distintos aspectos, dentro de los cuales sobresale la categoría que se refiere a las relaciones interpersonales, por cuanto es la que abarca la mayoría de aspectos que las estudiantes reportan como más significativos de la experiencia del trabajo integrado.

En cuanto a la relación con las compañeras de clase, las estudiantes valoran como significativo la oportunidad de trabajar con otras ellas, la importancia de apoyarse mutuamente y de trabajar en equipo, la relación construida a partir de conocerse mejor, pasar tiempo juntas, expresar sentimientos y dudas, compartir ideas, respetar opiniones, aprender unas de otras, entablar amistad y desarrollar compañerismo.

Los datos anteriores, coinciden con los resultados encontrados por Pereira (2010b) en una investigación en la que participaron estudiantes universitarias quienes igualmente destacaron la importancia de las relaciones interpersonales como parte de las dinámicas de aula; así como con los planteamientos de Gaff (1989), citado por Quintana (1998) y ellos citados por Mora (s. f.), quien señala que en el salón de clase todos y todas aportan en la construcción de los aprendizajes y que las relaciones interpersonales, justamente, promueven la cooperación entre estudiantes.

Con respecto a la interacción con las niñas y los niños de edad preescolar de las instituciones educativas en las que desarrollaron las investigaciones, las estudiantes destacaron como significativo la oportunidad de compartir de manera directa, trabajar y realizar actividades con ellas y ellos. De igual forma, hicieron referencia a las emociones y sentimientos que les generan sus 'caritas alegres', verlas y verlos disfrutar, sentir su cariño y amabilidad al recibirlas, así como la confianza que les tomaron.

En relación con la práctica docente y la inserción de las estudiantes en espacios educativos y de atención infantil es importante mencionar que algunas estudiantes expresaron que el trabajo en equipo les disminuyó el temor que genera enfrentarse por primera vez a un grupo de niñas y niños para trabajar con ellas y ellos, destacaron como significativo la oportunidad de visitar distintos contextos y centros educativos.

Al referirse a la elaboración del trabajo final, las estudiantes aludieron a la importancia de aprender la formalidad de cómo realizar un 'buen trabajo' para el futuro, saber cómo elaborar un trabajo de investigación, conocer sus partes y utilizar el formato APA. Otros comentarios se refirieron a la importancia de plantear un tema que resulte interesante, novedoso e importante, y otros mencionaron la importancia de aplicación de las técnicas grafico-plásticas y las técnicas musicales en los centros educativos. 
URL: http://www.una.ac.cr/educare

Tabla 3

Opiniones estudiantiles acerca de las dificultades enfrentadas en la actividad de integración curricular realizada en el I nivel de Diplomado

\begin{tabular}{|c|c|}
\hline \multicolumn{2}{|c|}{ 3. Categoría: Dificultades enfrentadas en la realización del trabajo integrado } \\
\hline Categorías emergentes & Aspectos \\
\hline Organización grupal & $\begin{array}{l}\text { - Conveniencia de horarios } \\
\text { - Definición de horarios de reunión } \\
\text { - Asistencia } \\
\text { - Puntualidad } \\
\text { - Coordinación a lo interno del grupo }\end{array}$ \\
\hline Elaboración del trabajo & $\begin{array}{l}\text { - Comprensión de las instrucciones } \\
\text { - Escogencia del tema } \\
\text { - Búsqueda de información } \\
\text { - Integración de las partes del trabajo } \\
\text { - Desarrollo de los capítulos } \\
\text { - Análisis de resultados }\end{array}$ \\
\hline Estilo de trabajo de las integrantes del grupo & $\begin{array}{l}\text { - Ritmo y tiempos de trabajo } \\
\text { - Actitud de compromiso } \\
\text { - Sentido de cooperación } \\
\text { - Sentido de responsabilidad } \\
\text { - Flexibilidad para aceptar y congeniar sus opiniones, comentarios, } \\
\text { críticas y puntos de vista }\end{array}$ \\
\hline
\end{tabular}

Dentro de los aspectos que representaron mayor dificultad para realizar el trabajo integrado, se destacan mediante la Tabla 3, principalmente aspectos de organización propios de todo trabajo grupal, tales como: ponerse de acuerdo para reunirse, coincidir en los horarios, la asistencia y la puntualidad a las sesiones de trabajo, así como la coordinación a lo interno del grupo. Otros aspectos se refirieron a la elaboración del trabajo mismo como comprender las instrucciones iniciales sobre lo que se solicitó, la escogencia del tema y la búsqueda de información, hasta el desarrollo del trabajo, la integración de sus partes, completar cada uno de los capítulos requeridos y finalmente analizar los resultados. También, les representó dificultad acoplarse al estilo de trabajo de cada una de las integrantes del grupo por las diferencias individuales en cuanto a ritmos y tiempos de trabajo, en algunos casos la falta de compromiso, poco sentido de cooperación y de responsabilidad mostrado por algunas de las integrantes del grupo, así como en algunos casos les fue difícil aceptar y congeniar sus opiniones, comentarios, críticas y puntos de vista.

A continuación, como resumen del análisis de datos efectuado anteriormente, se destacan mediante la tabla 4 y con apoyo de frecuencias y porcentajes, los aspectos sobresalientes a partir de los resultados obtenidos. 


\section{URL: http://www.una.ac.cr/educare}

Tabla 4

Principales resultados aportados por las estudiantes de Diplomado en Educación Preescolar acerca de la experiencia vivida con la actividad de integración curricular

\begin{tabular}{|c|c|l|}
\hline $\begin{array}{c}\text { Cantidad de } \\
\text { estudiantes }\end{array}$ & $\begin{array}{c}\text { Términos } \\
\text { porcentuales }\end{array}$ & \multicolumn{1}{|c|}{ Aspectos sobresalientes a partir de los resultados } \\
\hline 32 & $100 \%$ & $\begin{array}{l}\text { Valoraron positivamente la experiencia del trabajo realizado, tanto en el plano } \\
\text { académico, como en el personal. }\end{array}$ \\
\hline 27 & $84 \%$ & $\begin{array}{l}\text { Se refirieron a aspectos relativos a la interacción con las compañeras de } \\
\text { clase. }\end{array}$ \\
\hline 19 & $59 \%$ & $\begin{array}{l}\text { Se refirieron a aspectos relativos a la interacción con las niñas y los niños de } \\
\text { edad preescolar con quienes trabajaron. }\end{array}$ \\
\hline 11 & $34 \%$ & $\begin{array}{l}\text { Destacaron como significativo la oportunidad de conocer programas de } \\
\text { atención infantil como los CEN CINAI y los Hogares Comunitarios, en tanto } \\
\text { que la variedad de espacios les amplía su visión más allá de la realidad del } \\
\text { aula preescolar. }\end{array}$ \\
\hline 11 & $34 \%$ & $\begin{array}{l}\text { Aludieron a distintos aspectos relacionados propiamente con la elaboración } \\
\text { del trabajo final. }\end{array}$ \\
\hline 5 & $16 \%$ & $\begin{array}{l}\text { Identificaron como el aspecto más significativo, los aprendizajes construidos } \\
\text { a partir de la experiencia de integración, el hecho de que pudieron apreciar } \\
\text { cómo todos los aprendizajes construidos a lo largo del semestre se pueden } \\
\text { aplicar y presentar en un trabajo integrado, así como la posibilidad de ampliar } \\
\text { sus conocimientos a partir de investigar sobre el tema elegido. }\end{array}$ \\
\hline
\end{tabular}

\section{Conclusiones a partir del análisis de los resultados}

Es oportuno destacar que las valoraciones de las estudiantes del primer nivel de diplomado de la carrera de Licenciatura en Pedagogía con énfasis en Educación Preescolar fueron mayoritariamente positivas acerca de la experiencia de integración curricular desarrollada durante el I Semestre del 2011. Dichas valoraciones enfatizan aspectos referidos a la interacción tanto con las compañeras de clase, como con las niñas y los niños de edad preescolar, lo cual es indicativo de la importancia que tienen las relaciones interpersonales para las estudiantes desde el inicio de la carrera de Preescolar, aspecto que refiere a la importancia de los componentes personales, comunicativos y de interacción inherentes a los procesos pedagógicos y propios de aulas que priorizan el componente humanizador. Asimismo, se valora de forma significativa la oportunidad de familiarizarse con centros educativos y programas de atención infantil, lo cual reafirma a la práctica pedagógica la importancia de la inserción de estudiantes en espacios y contextos educativos desde este nivel de la carrera.

Es importante acotar que las estudiantes señalaron no solo aspectos cognoscitivos y personales, sino que también, se refirieron a la posibilidad de relacionar e integrar los contenidos de los cursos al abordar una temática de análisis investigativa. De igual manera, lo dicho por las estudiantes permite destacar la necesidad de que los distintos grupos de académicos y académicas se interesen por el desarrollo de diversas estrategias de integración curricular y que, mediante su contrastación, 
otros grupos de académicos y académicas se vean beneficiados y compartan las experiencias como una estrategia más para el verdadero análisis de la práctica profesional docente.

En cuanto a la integración de las áreas del currículo del nivel de preescolar, vale la pena subrayar que algunas estudiantes expresaron que el esfuerzo empeñado al intentar integrar todas las áreas las condujo a pensar de forma integrada. En términos pedagógicos, este aspecto es de suma importancia, pues refleja el propósito principal de la integración curricular, en tanto que logra superar la fragmentación del conocimiento desde el primer nivel de la carrera, lo cual es indispensable para la formación docente con visión holística e integral.

Lo antes mencionado refuerza, entonces, la idea de que enfocarse en la conformación de equipos de trabajo que permita al personal docente universitario diseñar y aplicar estrategias de integración curricular es un elemento que, desde la perspectiva estudiantil, se identifica como provechoso para su formación profesional.

Por último, cabe destacar que desde la visión de las estudiantes, surgen aspectos a considerar para mejorar en futuros trabajos de integración curricular por nivel. Para la elaboración de un trabajo final integrado, las estudiantes requieren indicaciones claras y consensuadas entre el cuerpo académico del nivel, esto implica no solo la coordinación entre docentes, sino que, además, permite tomar las previsiones necesarias para que los trabajos finales sean satisfactorios.

\section{Reflexiones finales, logros y retos}

Los procesos de integración curricular por niveles han de irse desarrollando de manera paulatina, para ello, es importante el conocimiento no solo de los académicos y las académicas que comparten un nivel, sino también, de los contenidos y objetivos que cada uno persigue en su propio curso e incluso de los que se persiguen para el nivel.

La integración curricular, como tal, implica la toma de conciencia de lo que cada quien hace en su curso, de las necesidades formativas de los estudiantes y las estudiantes, pero también, conlleva, un nivel de flexibilidad para poder abrir espacios para la integración, se esperaría que en la medida en que los equipos se vayan conociendo, respetando y acercándose a estrategias integrativas, en esa misma medida los procesos pueden tomar fluidez.

El recurrir a la comunicación vía correo electrónico en el caso particular del equipo del primer nivel de diplomado en Educación Preescolar fue una herramienta de agilización para la comunicación y la coordinación, aspecto que en este caso se subraya.

La experiencia, efectivamente, posibilitó la interacción respetuosa, basada en el diálogo e intercambio de ideas a un nivel académico. Además, se fortalecieron las relaciones interpersonales, en la medida en que se compartieron iniciativas, aportes y puntos de vista de docentes integrantes del equipo de trabajo.

Es importante señalar que, aunque cada uno de los cursos está bajo la responsabilidad directa del docente encargado, cuando se define que uno va a constituirse en eje integrador, desde todo punto de vista indica, que va a existir una responsabilidad directa del académico a cargo del mismo, pero ello no implica que se vaya a descuidar la participación responsable del resto de académicos y académicas de nivel. Más bien, lo que se espera es que desde cada uno de los cursos en los que está matriculado el estudiante y la estudiante, reciba aportes para el trabajo integrado que realizará. 
Para efectos de la elaboración de la investigación integrada, se designó el curso de Introducción a la investigación como el curso eje de integración del trabajo final. Es por ello, que, desde el programa de investigación, se fueron incorporando los aspectos que cada uno de los académicos de nivel esperaba encontrar en el informe final y para lo cual fueron brindando aportes a las estudiantes.

Asimismo, en cada uno de los programas de los otros cursos del nivel, se indicaron los elementos específicos de los contenidos del curso que deberían de estar presentes en el informe final de investigación, así como los aspectos a considerar en el rubro de evaluación. Sin duda alguna, todos estos aspectos de organización y establecimiento de criterios y lineamientos generales en los programas de los cursos ofrecieron mayor claridad a las estudiantes sobre el trabajo a desarrollar.

La evaluación de la investigación realizada por las estudiantes del nivel fue integral, ya que se unificaron criterios en el equipo de académicos y académicas para evaluar tanto el informe escrito, como la presentación oral de resultados. Ello se evidencia en el hecho de que, aunque cada académico y académica hizo una valoración de aspectos específicos de su propio curso, en el trabajo escrito y la exposición del mismo, la calificación final se promedió en una sola nota asignada por todos.

Sin embargo, lo anterior no es necesariamente indicativo de que en el nivel se dio una "evaluación como proceso integral, concertado, permanente, contextualizado y propositivo" referida a todos los aspectos curriculares. Queda claro, que el proceso de integración curricular se concretó en una actividad específica (trabajo de investigación integrado) y en el proceso que su elaboración requirió. Por tanto, no puede hablarse de 'integración curricular' como un proceso que engloba todos los aspectos y dimensiones curriculares de este nivel, pero sí se constituyó, tanto para docentes, como para estudiantes, en una valiosa experiencia en la búsqueda de la integración curricular, en este caso concreto, mediante el diseño, ejecución y evaluación conjunta de una investigación integrada por parte de las estudiantes.

El equipo de académicos y académicas del primer nivel de diplomado en Educación Preescolar logró crear un clima de solidaridad, compañerismo y empatía, lo cual, desde el primer momento, generó deseos de abrir espacios para buscar estrategias de integración curricular y hacer un esfuerzo por analizar de manera crítica la práctica docente, en busca de alternativas que permitieran la conformación como grupo de trabajo, deseoso de crecer como académicos y académicas, en pro de definir alternativas para el desarrollo integral de las estudiantes a nuestro cargo.

De igual manera, tal como lo indican los datos, las estudiantes participantes en la experiencia han destacado que la misma fue valiosa para su formación profesional y han recalcado no solo aspectos cognoscitivos, sino también personales y de interacción humana, lo cual, en nuestro criterio, es un importante elemento a considerar para futuras acciones en la búsqueda de la integración curricular.

Aunque es posible vislumbrar los logros que se han destacado anteriormente, son más los retos que quedan para promover procesos de integración curricular con mayor nivel de profundidad y alcance. En ese sentido y valorando lo realizado, es posible señalar como principal recomendación, que a nivel de la División de Educación Básica y del CIDE en general, se analice la posibilidad de darle continuidad a las experiencias de integración realizadas por los distintos grupos de académicos y académicas, promoviendo, para ello, la permanencia de los equipos de trabajo, en especial, con la consideración de que la constitución real de equipos involucra tiempo, conocimiento, disponibilidad, apertura, respeto, definición de metas comunes, solo para mencionar algunos aspectos. 
Vale la pena destacar que si en vez de permanencia lo que se da es la rotación del personal académico, entonces en cada semestre o cada año, lo que sucederá es una continua búsqueda para la constitución de equipos y alternativas para la integración curricular, desmereciéndose de esa manera, cualquier esfuerzo realizado por los equipos de trabajo por abocarse a un verdadero proceso de análisis crítico de la práctica docente y búsqueda responsable de posibilidades para su fortalecimiento y para el diseño y aplicación de estrategias de integración curricular.

Cualquier actividad de integración curricular debe reflejar un consenso por parte de los equipos de académicos y académicas en los distintos niveles de carrera y desde la coordinación de carrera, de manera que el proceso no responda únicamente a una política administrativa que se debe acatar, sino más bien, que sea el fruto de un proceso de reflexión sobre la práctica docente, que permita fortalecer humana y profesionalmente a los equipos constituidos y que sus frutos se expandan a ofrecer mejores oportunidades para el logro de una docencia exitosa, dentro de un marco de respeto a la diversidad, flexibilidad, coherencia y búsqueda de alternativas de trabajo en el aula cada vez más integradas.

El exponer esta experiencia, lleva como objetivo primordial, no solo el hacer un ejercicio de sistematización de la misma con miras a su fortalecimiento y mejora, sino también, compartir con la comunidad de académicos y académicas los logros obtenidos y promover de esa manera, una reflexión compartida sobre la práctica profesional docente, con la expectativa de que a futuro, se cuente con otras experiencias que quizá desde distinta óptica, permitan compartir estrategias diversas de integración curricular, que hagan cada día más interesante, esta convocatoria a la definición y aplicación de estrategias en este ámbito.

\section{Referencias bibliográficas}

Alfaro, M., Gamboa, A., Jiménez, S., Martín, J., Ramírez, A., Vargas, M. y Quirós, E. (2010). El equipo de investigación: un reto y una realidad. Revista Electrónica Educare, 14(1), 169-177. Recuperado de http://www.revistas.una.ac.cr/index.php/EDUCARE/article/view/1519

Beane, J. (2005). La integración del curriculum. Madrid: Ediciones Morata.

Centro de Investigación y Docencia en Educación (CIDE). (2010). Oficio CIDE-VD-281-2010. Heredia, Costa Rica: Decanatura.

División de Educación Básica. Centro de Investigación y docencia en Educación [CIDE]. (2008). Plan de estudios de la carrera de bachillerato en Pedagogía con énfasis en Educación Preescolar con salida lateral de diplomado. Heredia, Costa Rica: CIDE-UNA.

Esquivel, L., Induni, G., Madrigal, V., Pereira, Z., Solano, J. y Umaña, W. (2004). Conformación de un equipo de investigadores: Una experiencia concreta. Revista Electrónica Educare, 6, 193204. Recuperado de http://www.revistas.una.ac.cr/index.php/EDUCARE/article/view/1088

Mora, R. M. (s. f.). Integración curricular. Recuperado de http://morasolano.tripod.com/id15.html 
Pereira, Z. (2010a) Las dinámicas interactivas en el ámbito universitario: el clima de aula. Revista Electrónica Educare, 14(Extraordinario), 7-20. Recuperado de http://www.revistas.una.ac.cr/ index.php/EDUCARE/article/view/1524

Pereira, Z. (2010b). La mirada de estudiantes de la Universidad Nacional hacia el docente y la docente: Sus características y clima de aula. Revista Electrónica Educare,14(Extraordinario), 21-39. Recuperado de http://www.revistas.una.ac.cr/index.php/EDUCARE/article/view/1525/1445

Sánchez, J. (s. f.). Integración curricular de las TICs: Conceptos e ideas. Recuperado de http:// www.educarenpobreza.cl/UserFiles/P0001/Image/gestion portada/documentos/CD-24 Doc. integracion curriculartic (ficha 17).pdf

Torres, J. (2006). Globalización e Interdisciplinariedad: El curriculum integrado (5 ${ }^{\mathrm{a}}$ ed., reimpresión). Madrid: Ediciones Morata.

Universidad Nacional. Costa Rica [UNA]. (s. f.). Áreas estratégicas: Docencia. Recuperado de http://www.una.ac.cr//index.php?option=com_content\&task=view\&id=244

Universidad Nacional, Costa Rica. [UNA]. (2008). Modelo Pedagógico. Recuperado de http:// unaweb.una.ac.cr/index.php?option=com remository\&Itemid=0\&func $=$ startdown \&id=141

Zúñiga, I. y López, L. (2003). Guía para la elaboración y presentación de informes de trabajos finales de graduación y de cursos. Heredia: Universidad Nacional, Centro de Investigación y Docencia en Educación. 